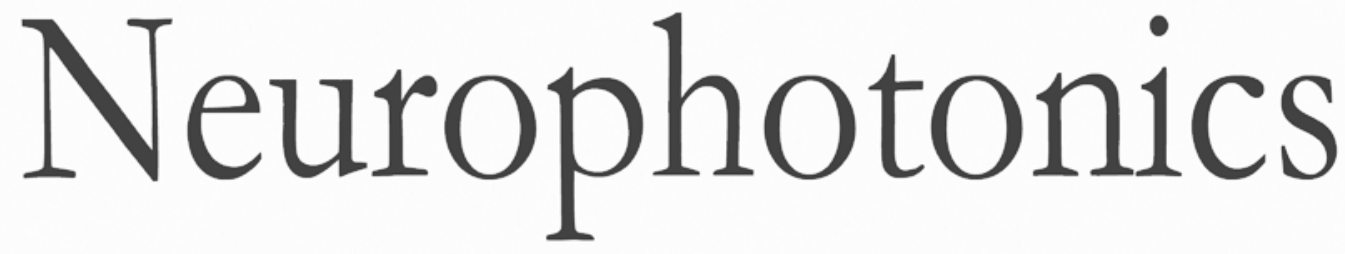

\title{
Two-photon microscopy measurement of cerebral metabolic rate of oxygen using periarteriolar oxygen concentration gradients
}

Sava Sakadžić

Mohammad A. Yaseen

Rajeshwer Jaswal

Emmanuel Roussakis

Anders M. Dale

Richard B. Buxton

Sergei A. Vinogradov

David A. Boas

Anna Devor

Sava Sakadžić, Mohammad A. Yaseen, Rajeshwer Jaswal, Emmanuel Roussakis, Anders M. Dale, Richard B. Buxton, Sergei A. Vinogradov, David A. Boas, Anna Devor, "Two-photon microscopy measurement of cerebral metabolic rate of oxygen using periarteriolar oxygen concentration gradients,"

Neurophoton. 3(4), 045005 (2016), doi: 10.1117/1.NPh.3.4.045005. 


\title{
Two-photon microscopy measurement of cerebral metabolic rate of oxygen using periarteriolar oxygen concentration gradients
}

\author{
Sava Sakadžić, ${ }^{a, \star}$ Mohammad A. Yaseen, ${ }^{a}$ Rajeshwer Jaswal, ${ }^{a}$ Emmanuel Roussakis, ${ }^{b}$ Anders M. Dale, ${ }^{c, d}$ \\ Richard B. Buxton, ${ }^{d}$ Sergei A. Vinogradov, ${ }^{\mathrm{b}}$ David A. Boas, ${ }^{\mathrm{a}}$ and Anna Devor ${ }^{\mathrm{a}, \mathrm{c}, \mathrm{d}}$ \\ ${ }^{a}$ Massachusetts General Hospital and Harvard Medical School, Optics Division, MGH/HMS/MIT Athinoula A. Martinos Center for Biomedical \\ Imaging, Department of Radiology, 149 13th Street, Charlestown, Massachusetts 02129, United States \\ bUniversity of Pennsylvania, Departments of Biochemistry and Biophysics and Chemistry, 422 Curie Boulevard, Philadelphia, Pennsylvania 19104, \\ United States \\ 'University of California San Diego, Department of Neurosciences, 9500 Gilman Drive, La Jolla, California 92093, United States \\ ¿University of California San Diego, Department of Radiology, 9500 Gilman Drive, La Jolla, California 92093, United States
}

\begin{abstract}
The cerebral metabolic rate of oxygen $\left(\mathrm{CMRO}_{2}\right)$ is an essential parameter for evaluating brain function and pathophysiology. However, the currently available approaches for quantifying $\mathrm{CMRO}_{2}$ rely on complex multimodal imaging and mathematical modeling. Here, we introduce a method that allows estimation of $\mathrm{CMRO}_{2}$ based on a single measurement modality - two-photon imaging of the partial pressure of oxygen $\left(\mathrm{PO}_{2}\right)$ in cortical tissue. We employed two-photon phosphorescence lifetime microscopy (2PLM) and the oxygen-sensitive nanoprobe PtP-C343 to map the tissue $\mathrm{PO}_{2}$ distribution around cortical penetrating arterioles. $\mathrm{CMRO}_{2}$ is subsequently estimated by fitting the changes of tissue $\mathrm{PO}_{2}$ around arterioles with the Krogh cylinder model of oxygen diffusion. We measured the baseline $\mathrm{CMRO}_{2}$ in anesthetized rats and modulated tissue $\mathrm{PO}_{2}$ levels by manipulating the depth of anesthesia. This method provides $\mathrm{CMRO}_{2}$ measurements localized within $\sim 200 \mu \mathrm{m}$ and it may provide oxygen consumption measurements in individual cortical layers or within confined cortical regions, such as in ischemic penumbra and the foci of functional activation. $\odot 2016$ Society of Photo-Optical Instrumentation Engineers (SPIE) [DOI: 10.1117/1.NPh.3.4.045005]
\end{abstract}

Keywords: oxygen metabolism; oxygen partial pressure; two-photon microscopy; phosphorescence; cerebral cortex. Paper 16023R received Apr. 23, 2016; accepted for publication Sep. 16, 2016; published online Oct. 17, 2016.

\section{Introduction}

Estimation of the cerebral metabolic rate of oxygen $\left(\mathrm{CMRO}_{2}\right)$ is a challenging task that in common practice requires knowledge of both blood oxygenation and flow. ${ }^{1} \mathrm{CMRO}_{2}$ is estimated from multiple measurements analyzed within the context of an appropriate mathematical model of the physiology and the measured quantities. Indeed, all methods of estimating $\mathrm{CMRO}_{2}$ are essentially solving a mass balance equation, where $\mathrm{CMRO}_{2}$ is equated to the difference of oxygen flowing into a region of interest and the oxygen flowing out. Such modeling adds a layer of complexity to estimating $\mathrm{CMRO}_{2}$ and raises concerns about the accuracy of the estimates due to the assumptions and limitations of the experimental methods and models used.

Several methods exist to measure $\mathrm{CMRO}_{2}$ in humans and in small animals. The leading method for measuring $\mathrm{CMRO}_{2}$ in humans is positron emission tomography (PET) using ${ }^{15} \mathrm{O}$-labeled oxygen and water to estimate oxygen uptake and cerebral blood flow (CBF), respectively. ${ }^{2,3}$ Another method gaining popularity in human studies is the "calibrated" blood oxygenation level dependent (BOLD) functional magnetic resonance imaging (fMRI) approach. ${ }^{4-7}$ This method combines BOLD fMRI with arterial spin labeling methods and includes an additional calibration step (e.g., measuring local CBF and BOLD responses to mild hypercapnia). ${ }^{1}$ Near-infrared spectroscopy measurement of cerebral blood oxygenation is used in

*Address all correspondence to: Sava Sakadžić, E-mail: sava.sakadzic@mgh. harvard.edu combination with blood flow measurements to assess $\mathrm{CMRO}_{2}$ in both adults and neonates. ${ }^{8,9}$ Finally, magnetic resonance spectroscopy (MRS) based on ${ }^{31} \mathrm{P}$ and ${ }^{31} \mathrm{C}$ can be used in humans to assess metabolic parameters tightly coupled with $\mathrm{CMRO}_{2}$, such as the tricarboxylic acid cycle rate and cerebral metabolic rate of adenosine triphosphate $\left(\mathrm{CMR}_{\mathrm{ATP}}\right)$, respectively. ${ }^{10,11}$ In animals, $\mathrm{CMRO}_{2}$ is also often estimated using a combination of measurements of blood oxygenation and flow. PET and fMRI techniques were appropriately modified to allow high spatial resolution measurements of $\mathrm{CMRO}_{2}$ (e.g., $\sim 1.2 \mathrm{~mm}$ for microPET ${ }^{12}$ and voxel size of several $\mathrm{mm}^{3}$ for MRI ${ }^{13,14}$ ). In addition, MRS can be used to assess $\mathrm{CMRO}_{2}$ directly based on ${ }^{17} \mathrm{O}$ with a few millimeters spatial resolution. ${ }^{15,16}$ Optical imaging methods offer numerous choices to measure $\mathrm{CMRO}_{2}$ in animals by combining blood oxygenation and flow measurements. Blood oxygenation can be assessed by spectroscopic optical imaging of hemoglobin saturation by using intrinsic signals, ${ }^{17-20}$ visible optical coherence tomography (OCT), ${ }^{21,22}$ photoacoustic imaging (PAI), ${ }^{23,24}$ or phosphorescence lifetime imaging. ${ }^{25-28} \mathrm{CBF}$ can be obtained by laser Doppler or speckle contrast imaging, ${ }^{19,29}$ OCT, ${ }^{30,31}$ or PAI. ${ }^{23,24}$ Due to the complex cortical microvascular morphology (i.e., no single vascular input and output in the cortex), in cases where $\mathrm{CBF}$ and oxygenation were measured in individual vessels, both parameters must be acquired over a large field of view $\left(\sim 1 \mathrm{~mm}\right.$ or larger) in order to estimate $\mathrm{CMRO}_{2}$. By assuming the cortical thickness and that no vessels assessed

2329-423X/2016/\$25.00 @ 2016 SPIE 
on the cortical surface supply subcortical regions, the estimated $\mathrm{CMRO}_{2}$ represents an average over the entire cortical depth and field of view.

Here, we present a high-spatial-resolution and more direct method for estimating $\mathrm{CMRO}_{2}$ that relies on a single experimentally obtained parameter-the partial pressure of oxygen $\left(\mathrm{PO}_{2}\right)$ in tissue in the immediate vicinity $(<200 \mu \mathrm{m})$ of penetrating cortical arterioles. Earlier attempts to measure $\mathrm{CMRO}_{2}$ by directly fitting the solution of Poisson's equation to $\mathrm{PO}_{2}$ around vessels were limited by the inability to measure tissue $\mathrm{PO}_{2}$ at depth with adequate sensitivity and resolution. ${ }^{32}$ This limitation was recently overcome by the development of $2 \mathrm{PLM}$ of $\mathrm{PO}_{2}$ that allows measurements of both intravascular and interstitial (tissue) cortical $\mathrm{PO}_{2}$ in vivo with high spatial and temporal resolution. ${ }^{33-42}$ Here, we estimated $\mathrm{CMRO}_{2}$ by applying the Krogh cylinder model ${ }^{43}$ to periarteriolar tissue around penetrating arterioles and fitting the experimentally determined distributions of tissue $\mathrm{PO}_{2}$ to the Krogh-Erlang solution. Our method provides $\mathrm{CMRO}_{2}$ measurements localized within $\sim 200 \mu \mathrm{m}$, which is defined by the tissue area where $\mathrm{PO}_{2}$ distributions are measured, and it can be easily combined with other optical microscopy tools for preclinical studies of cerebral function and metabolism.

\section{Methods}

\subsection{Animal Preparation}

Sprague Dawley rats (250 to $320 \mathrm{~g}$ ) were anesthetized with isoflurane (1.5\% to $2 \%$ in a mixture of $\mathrm{O}_{2}$ and air), temperature controlled, tracheotomized, and catheters were inserted in the femoral artery and vein for administering the anesthesia and dyes, and for measuring blood gases, $\mathrm{pH}$, and blood pressure. We created a cranial window in the center of the parietal bone with the dura removed. Before sealing the window, we pressureinjected $\approx 0.1 \mu \mathrm{L}$ of PtP-C343 $\left(1.4 \times 10^{-4} \mathrm{M}\right) \sim 300 \mu \mathrm{m}$ below the surface of the brain using a glass micropipette. The imaging was performed a few hundred microns from the injection site. Slow diffusion of the probe in the bulk-loaded brain tissue allows imaging of $\mathrm{PO}_{2}$ for several hours following the injection. During the measurements, we ventilated rats with a mixture of air and oxygen adjusting the fraction of the inspired oxygen $\left(\mathrm{FiO}_{2}=21 \%\right.$ to $\left.24 \%\right)$ to maintain systemic arterial $\mathrm{PO}_{2}$ at 95 to $110 \mathrm{mmHg}$. Isoflurane was discontinued and anesthesia maintained with a $50 \mathrm{mg} / \mathrm{kg}$ intravenous bolus of alpha-chloralose followed by continuous intravenous infusion at $40 \mathrm{mg} /(\mathrm{kg} \mathrm{h})$. The systemic arterial blood $\mathrm{PCO}_{2}$ was 35 to $44 \mathrm{mmHg}$ and $\mathrm{pH}$ was 7.35 to 7.42. All experimental procedures were approved by the Massachusetts General Hospital Sub-Committee on Research Animal Care.

\subsection{Experimental Protocol}

Two-photon in vivo brain imaging was performed by using our previously described custom-built microscope, controlled by the custom-designed software. ${ }^{34,44}$ The optical beam was scanned in the $X Y$ plane by galvanometer scanners and focused on the sample by an objective (Olympus 20X XLumPlanFL; NA $=0.95$ ). A motorized stage controlled the focal position along the vertical axis $(Z)$, and an electro-optic modulator served to gate the output of the high-repetition rate pulsed laser (Mai Tai, Spectra Physics).
We excited PtP-C343 phosphorescence by $10-\mu$ s-long trains of femtosecond pulses at $840 \mathrm{~nm}$, followed by a $290-\mu$ s-long phosphorescence collection period. The emission was detected by two of the four photomultiplier tubes (PMTs) in our detector array. The phosphorescence output was detected by a photoncounting PMT module (H10770PA-50, Hamamatsu). In a typical experiment, we performed phosphorescence detection in two steps. First, we raster scanned the excitation beam over the field of view, rendering two-dimensional survey maps of the integrated emission intensity. After mapping the distribution of intensity, we averaged 500 to 2000 phosphorescence decays in selected point locations in the tissue for accurate $\mathrm{PO}_{2}$ determination. This acquisition time corresponded to a temporal resolution of 0.16 to $0.76 \mathrm{~s}$ per single-point $\mathrm{PO}_{2}$ measurement. $\mathrm{PO}_{2}$ data were typically collected in a grid pattern spanning $\sim 300 \times$ $300 \mu \mathrm{m}^{2}$ and consisting of $\approx 500$ points, which, together with the survey mapping and selection of the grid points, took 2 to $5 \mathrm{~min}$ to collect. Finally, we converted phosphorescence lifetimes into $\mathrm{PO}_{2}$ values by using Stern-Volmer calibration plots. ${ }^{33}$

Structural images of the cortical vasculature were obtained by imaging the blood plasma labeled with fluorescein isothiocyanate (FITC)-dextran. We collected images of the vasculature at the $\mathrm{PO}_{2}$ imaging planes immediately before or after $\mathrm{PO}_{2}$ imaging. These images were coregistered with the $\mathrm{PO}_{2}$ data and used to define blood vessel boundaries. We also collected a three-dimensional vascular angiogram at the end of each experiment.

\subsection{Data Processing}

We assumed that oxygen diffusion from a penetrating arteriole can be approximated by the Krogh cylinder model of oxygen diffusion from a vessel. ${ }^{43}$ In the Krogh cylinder model [Fig. 1(a)], a vessel with cylindrical shape and radius $R_{\text {art }}$ supplies a tissue cylinder with radius $R_{\mathrm{t}}$. We also assumed that oxygen consumption $\left(\mathrm{CMRO}_{2}\right)$, tissue oxygen diffusivity $(D)$, and solubility $(\alpha)$ constants are spatially homogeneous and temporally invariant. If $D$ is isotropic and all important microvascular oxygen transport phenomena are steady state, the oxygen diffusion in a tissue satisfies Poisson's equation:

$\Delta \mathrm{PO}_{2}(\vec{r})=\frac{\mathrm{CMRO}_{2}}{D \alpha}$,

where $\Delta$ is the Laplace operator and $\mathrm{PO}_{2}(\vec{r})$ is the oxygen partial pressure at location $\vec{r}$, respectively. By assuming that (1) axial oxygen diffusion is insignificant, (2) $\partial \mathrm{PO}_{2}(r) / \partial r=0$ at the tissue cylinder boundary $R_{\mathrm{t}}$, and (3) $\mathrm{PO}_{2}$ in tissue at the arteriolar wall $\left[\mathrm{PO}_{2}\left(R_{\text {art }}\right)\right]$ is labeled $\mathrm{PO}_{2 \text {,art }}$, the solution of Eq. (1) can be expressed as a well-known Krogh-Erlang formula describing oxygen diffusion from a cylinder: ${ }^{43}$

$$
\begin{aligned}
\mathrm{PO}_{2}(r)= & \mathrm{PO}_{2, \text { art }}+\frac{\mathrm{CMRO}_{2}}{4 D \alpha}\left(r^{2}-R_{\text {art }}^{2}\right) \\
& -\frac{\mathrm{CMRO}_{2}}{2 D \alpha} R_{\mathrm{t}}^{2} \ln \left(\frac{r}{R_{\text {art }}}\right)
\end{aligned}
$$

with a difference that in Eq. (2) we consider a tissue cylinder around the penetrating cortical arteriole instead of the originally considered tissue cylinder around a capillary.

In our experiments, $R_{\text {art }}$ was estimated from the vascular anatomical images obtained by two-photon microscopy of FITC-labeled blood plasma. We assumed the tissue oxygen 
(a)
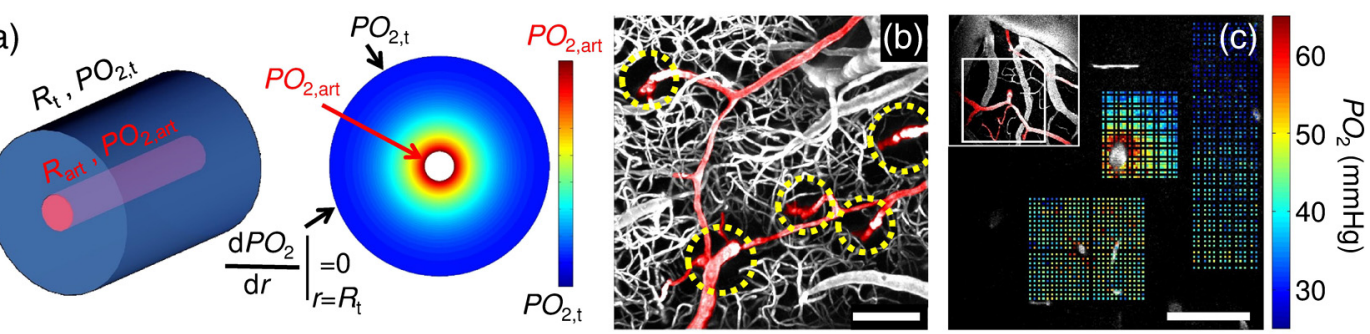

Fig. 1 The Krogh cylinder, cortical vascular morphology, and tissue $\mathrm{PO}_{2}$. (a) The Krogh cylinder model of oxygen diffusion from a vessel. An infinitely long tissue cylinder with radius $R_{\mathrm{t}}$ is supplied by an infinitely long arteriole with radius $R_{\text {art }} . \mathrm{PO}_{2}$ map on the right hand side is computed based on Krogh-Erlang equation [Eq. (2)]. The zero oxygen flux boundary condition $\left(d \mathrm{PO}_{2} / d r=0\right.$ at $\left.r=R_{\mathrm{t}}\right)$ is satisfied at the external tissue boundary. (b) Maximum intensity projection of a 500- $\mu$ m-thick microvascular stack. Pial artery and adjacent diving arterioles are colored red. Yellow circles emphasize capillaryfree spaces around diving arterioles. Scale bar, $100 \mu \mathrm{m}$. (c) Baseline tissue $\mathrm{PO}_{2}$ map (color coded), overlaid on a FITC image of the microvasculature $100 \mu \mathrm{m}$ below the brain surface. Inset in upper left corner shows $200-\mu \mathrm{m}$-thick MIP of FITC-labeled microvasculature. Arterioles are colored red. White rectangle in inset outlines the position of the panel (c). Scale bar, $100 \mu \mathrm{m}$.

permeability $D \alpha$ to be equal to the oxygen permeability of water $\left.\left(\alpha=1.39 \mu \mathrm{M} \mathrm{mmHg}^{-1} ; D=4 \times 10^{-5} \mathrm{~cm}^{2} \mathrm{~s}^{-1}\right)\right)^{45}$ Periarteriolar tissue $\mathrm{PO}_{2}$ measurements were coregistered with the vascular anatomical images. The radial distance from the center of the penetrating arteriole $r$ was estimated for each measured tissue $\mathrm{PO}_{2}$ point. Equation (2) was fit for $\mathrm{CMRO}_{2}, R_{\mathrm{t}}$, and $\mathrm{PO}_{2, \text { art }}$. Nonlinear least squares fitting was performed by the Levenberg-Marquardt algorithm. Since the Krogh cylinder model does not include tissue regions beyond radius $R_{\mathrm{t}}$, in each iterative step of the fitting procedure, the sum of the squares of residuals was calculated only for the measured $\mathrm{PO}_{2}$ points up to a radial distance of $R_{\mathrm{t}}$. All the data processing procedures were custom-written in MATLAB (MathWorks Inc.).

\section{Results}

The cortical vasculature consists of a planar mesh of pial arteries and veins at the cortical surface that dive into the cortex supplying a complex microvascular network and draining the blood back to the surface. Figure 1(b) shows a typical cortical vascular anatomical image of the rat cortex. Vascular images, such as in Fig. 1(b), reveal that periarteriolar tissue around penetrating arterioles is largely devoid of capillaries, whereas the tissue away from penetrating arterioles exhibits high capillary density. One possible explanation for this morphological feature could be that the periarteriolar region is characterized by reduced cell density, and thus, it does not need as much oxygen as other tissue regions. However, published data on the density of neurons and astrocytes in cerebral cortex demonstrate the absence of anatomical differences between periarteriolar spaces and other cortical regions. ${ }^{46}$ Therefore, a more likely explanation is that highly oxygenated blood from cortical penetrating arterioles efficiently supplies large periarteriolar tissue territories, and formation of capillaries near the arterioles is simply not required. Figure 1(c) shows an example map of cortical tissue oxygen concentration, where large $\mathrm{PO}_{2}$ gradients originating from the diving arteriole are dominating the tissue $\mathrm{PO}_{2}$ landscape. The central part of Fig. 1(c) shows a typical radially symmetrical profile of periarteriolar tissue $\mathrm{PO}_{2}$ in a plane parallel with the cortical surface (e.g., perpendicular to the propagation axis of the penetrating arteriole).

To obtain data for the $\mathrm{CMRO}_{2}$ fitting, we typically imaged tissue $\mathrm{PO}_{2}$ over a grid spanning $\sim 300 \times 300 \mu \mathrm{m}^{2}$ and selected a subset of the $\mathrm{PO}_{2}$ data within an arc around the arteriole with the most uniform and steepest $\mathrm{PO}_{2}$ descent. Figure 2 shows four individual examples, where $\mathrm{CMRO}_{2}$ was estimated based on periarteriolar tissue $\mathrm{PO}_{2}$ profiles from the upper 100 to $160 \mu \mathrm{m}$ of cortex in four different subjects. The estimated mean $\mathrm{CMRO}_{2}$ was $1.71 \pm 0.16 \mu \mathrm{mol} \mathrm{cm}^{-3} \mathrm{~min}^{-1}$, where individual measurements varied between $1.4 \mu \mathrm{mol} \mathrm{cm}^{-3} \mathrm{~min}^{-1}$ and $2.1 \mu \mathrm{mol} \mathrm{cm}^{-3} \mathrm{~min}^{-1}$ (Table 1), in agreement with the previously measured $2.5 \pm 1.0 \mu \mathrm{mol} \mathrm{cm}{ }^{-3} \mathrm{~min}^{-1}$ in rats under a similar anesthesia regime. ${ }^{47}$ The estimated Krogh cylinder tissue radius $R_{\mathrm{t}}$ varied between 70.4 and $106.5 \mu \mathrm{m}$, in agreement with the measured distances $R_{\text {cap,min }}$ of the closest capillaries to the penetrating arteriole (Table 1).

Figure 3 illustrates the likely influence of physiological and morphological parameters, such as blood pressure, heart rate, and microvascular configuration upon cerebral $\mathrm{PO}_{2}$ and estimation of $\mathrm{CMRO}_{2}$. Two $\mathrm{CMRO}_{2}$ measurements were performed along the same penetrating arteriole in rat II (Fig. 2) at two imaging depths (150 and $130 \mu \mathrm{m}$ below the cortical surface). The data at the two depths were collected $30 \mathrm{~min}$ apart. The result indicates a significant increase in the baseline tissue $\mathrm{PO}_{2}$ in the second measurement in comparison with the first measurement. This is likely due to an increase in blood flow, since no noticeable change in $\mathrm{PO}_{2}$ was previously observed in penetrating arterioles of similar caliber over the difference in depth of only $20 \mu \mathrm{m} .{ }^{41,42}$ The variation of the baseline tissue $\mathrm{PO}_{2}$ and change in microvascular configuration at different depths may influence the $\mathrm{CMRO}_{2}$ fitting procedure. However, we anticipated that $\mathrm{CMRO}_{2}$ should not exhibit significant changes within a cortical layer. In agreement with this expectation, the estimated $\mathrm{CMRO}_{2}$ at these two depths was found to be the same (1.4 $\mu \mathrm{mol} \mathrm{cm}^{-3} \mathrm{~min}^{-1}$; Table 2).

To experimentally manipulate $\mathrm{CMRO}_{2}$, we modulated the level of anesthesia in rat IV (Fig. 2) by applying isoflurane (2\%) on top of the ongoing alpha-chloralose anesthesia. Adding isoflurane resulted in a decrease in both blood pressure and heart rate (from 100 to $76 \mathrm{mmHg}$ and from 8 to $6.5 \mathrm{~Hz}$, respectively) and an increase in tissue $\mathrm{PO}_{2}$. The increase in $\mathrm{PO}_{2}$ is expected if neuronal activity and the associated $\mathrm{CMRO}_{2}$ decrease under deeper anesthesia. Isoflurane is also a vasodilator that increases blood perfusion, which further reduces oxygen extraction fraction and increases tissue $\mathrm{PO}_{2}{ }^{48}$ Figure 4 shows the comparison of $\mathrm{CMRO}_{2}$ estimated 
(a)
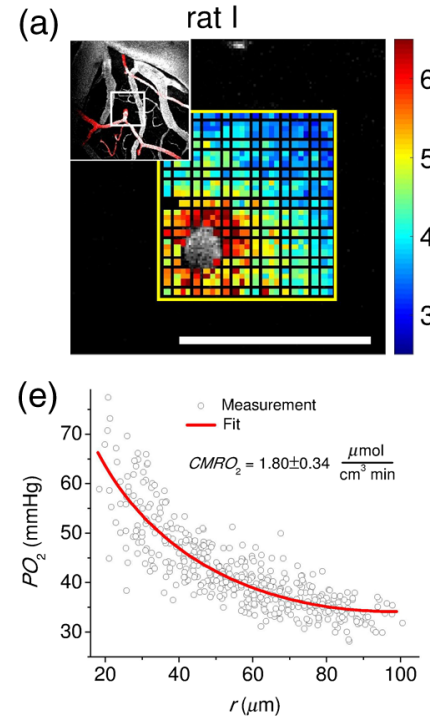

(b)

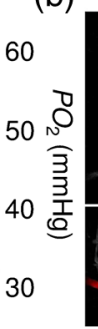

(f)

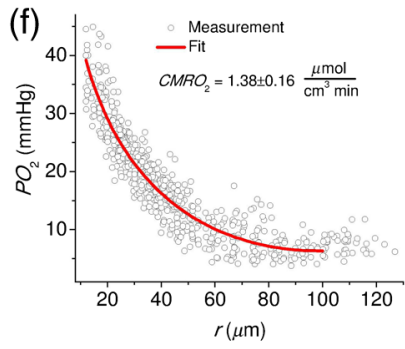

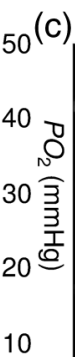

(g)

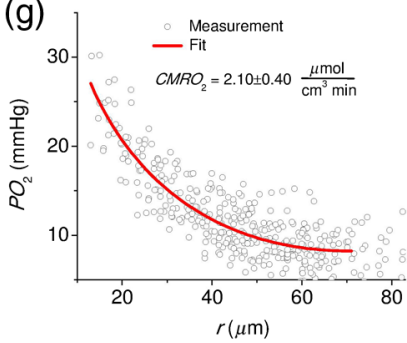

rat IV
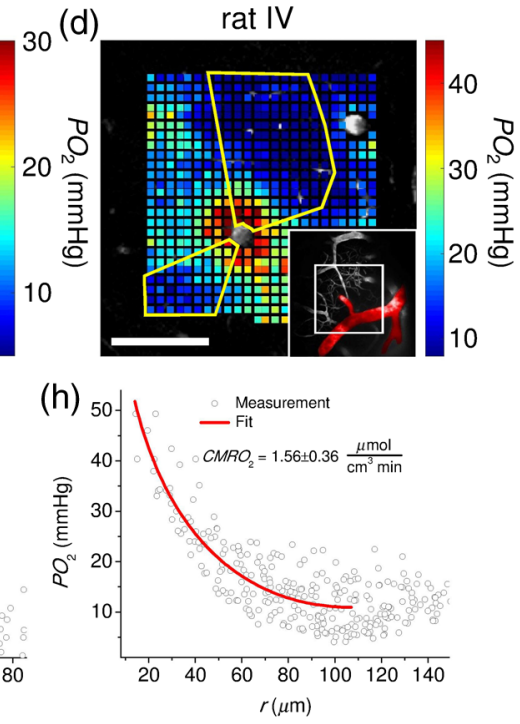

Fig. 2 Baseline $\mathrm{CMRO}_{2}$ measurements. (a-d) Tissue $\mathrm{PO}_{2}$ maps (color coded) around penetrating arterioles overlaid on the corresponding FITC images of microvasculature in different animals (rats I to IV). Insets show MIPs of FITC-labeled microvasculature. Arterioles in insets are colored red. The white rectangle in each insert outlines the position of the corresponding panel with the $\mathrm{PO}_{2}$ data. Yellow lines outline regions of interest with $\mathrm{PO}_{2}$ data included in the fitting procedure. Imaging depths below brain surface are $100 \mu \mathrm{m}$ (rat I), $150 \mu \mathrm{m}$ (rat II), $124 \mu \mathrm{m}$ (rat III), and $160 \mu \mathrm{m}$ (rat IV). Scale bars, $100 \mu \mathrm{m}$. (e-h) Tissue $\mathrm{PO}_{2}$ from the corresponding upper panels as a function of the radial distance from the penetrating arteriole with $\mathrm{PO}_{2}$ fit indicated by solid line.

Table 1 Estimated Krogh cylinder parameters.

\begin{tabular}{|c|c|c|c|c|c|}
\hline Rat \# & $\mathrm{CMRO}_{2}\left(\mu \mathrm{mol} \mathrm{cm}^{-3} \mathrm{~min}^{-1}\right)$ fitted & $R_{\mathrm{t}}(\mu \mathrm{m})$ fitted & $\mathrm{PO}_{2, \text { art }}(\mathrm{mmHg})$ fitted & $R_{\text {art }}(\mu \mathrm{m})$ fixed & $R_{\text {cap } \text { min }}(\mu \mathrm{m})$ \\
\hline 1 & $1.80 \pm 0.34^{\mathrm{a}}$ & $98.8 \pm 6.8$ & $79.9 \pm 1.7$ & 10.6 & 111 \\
\hline II & $1.38 \pm 0.16$ & $99.1 \pm 4.5$ & $48.7 \pm 0.6$ & 7.5 & 86 \\
\hline III & $2.10 \pm 0.40$ & $70.4 \pm 4.7$ & $35.4 \pm 1.1$ & 7.5 & 69 \\
\hline IV & $1.56 \pm 0.36$ & $106.5 \pm 8.9$ & $63.1 \pm 2.8$ & 9.1 & 70 \\
\hline
\end{tabular}

aStandard error.
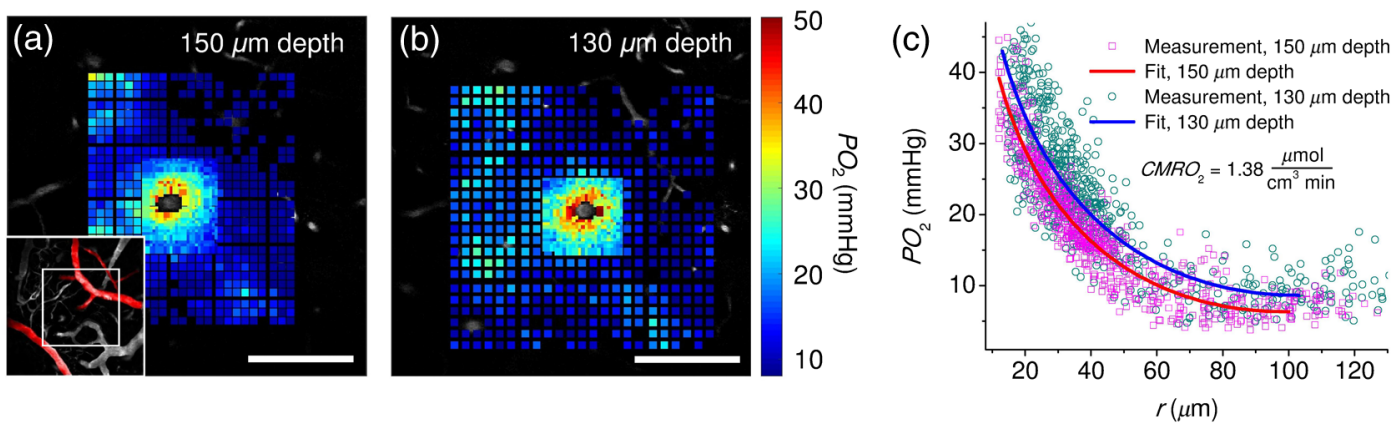

Fig. $3 \mathrm{CMRO}_{2}$ estimation at different baseline tissue $\mathrm{PO}_{2}$ and two cortical depths along the same penetrating arteriole. ( $\mathrm{a}, \mathrm{b})$ Tissue $\mathrm{PO}_{2}$ maps (color coded) overlaid on FITC images of microvasculature at depths of (a) $150 \mu \mathrm{m}$ and (b) $130 \mu \mathrm{m}$ below the brain surface. Inset in the lower left corner shows $160-\mu \mathrm{m}$-thick MIPs of FITC-labeled microvasculature. Arterioles are colored red. White rectangles in insets outline the position of the panels (a) and (b). Regions of interest with $\mathrm{PO}_{2}$ data included in the fitting procedure are the same as in rat II (Fig. 2). Scale bars, $100 \mu \mathrm{m}$. (c) Tissue $\mathrm{PO}_{2}$ dependence on the radial distance from the arteriole with $\mathrm{PO}_{2}$ fits indicated by solid lines. 
Table 2 Estimated Krogh cylinder parameters at different depths and baseline tissue $\mathrm{PO}_{2}$.

\begin{tabular}{lcccc} 
Depth $(\mu \mathrm{m})$ & $\mathrm{CMRO}_{2}\left(\mu \mathrm{mol} \mathrm{cm}^{-3} \mathrm{~min}^{-1}\right)$ fitted & $R_{\mathrm{t}}(\mu \mathrm{m})$ fitted & $\mathrm{PO}_{2, \text { art }}(\mathrm{mmHg})$ fitted & $48.7 \pm 0.6$ \\
150 & $1.38 \pm 0.16^{\mathrm{a}}$ & $99.1 \pm 4.5$ & $R_{\text {art }}(\mu \mathrm{m})$ fixed \\
130 & $1.38 \pm 0.26$ & $102.7 \pm 7.8$ & $54.9 \pm 1.2$ & 7.5 \\
\hline
\end{tabular}

${ }^{\text {a }}$ Standard error.
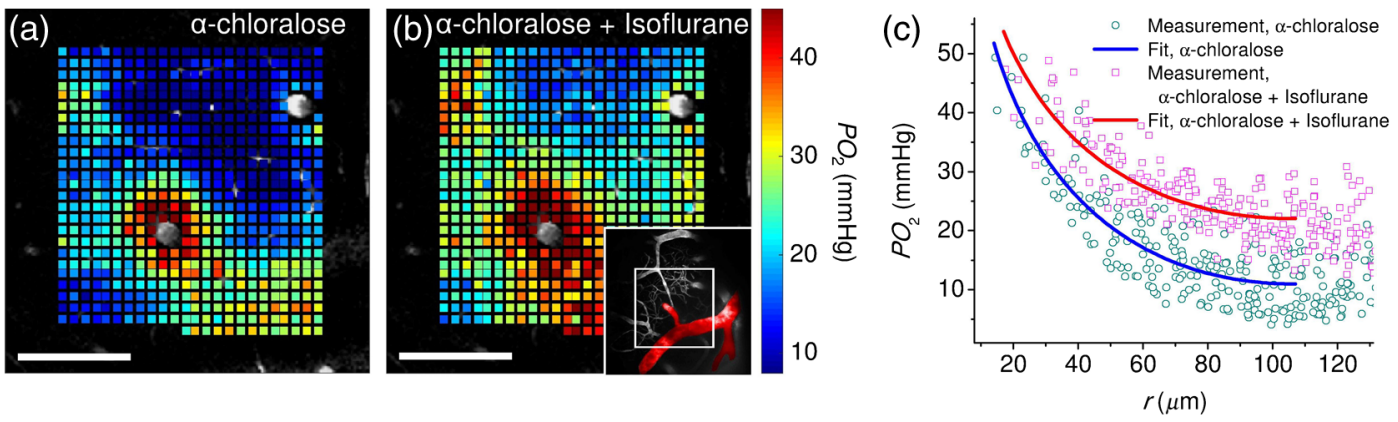

Fig. $4 \mathrm{CMRO}_{2}$ estimation at two anesthesia depths. (a, b) Tissue $\mathrm{PO}_{2}$ maps (color coded) at two different anesthesia levels, overlaid on FITC image of microvasculature $160 \mu \mathrm{m}$ below brain surface. (a) Alphachloralose anesthesia; (b) combined alpha-chloralose and isoflurane anesthesia. Inset in lower right corner shows 312- $\mu \mathrm{m}$-thick MIP of FITC-labeled microvasculature. Arterioles are colored red. White rectangle in inset outlines the positions of the panels (a) and (b). Regions of interest with $\mathrm{PO}_{2}$ data included in the fitting procedure are the same as in rat IV (Fig. 2). Scale bars, $100 \mu \mathrm{m}$. (c) Tissue $\mathrm{PO}_{2}$ dependence on the radial distance from the arteriole with $\mathrm{PO}_{2}$ fits indicated by solid lines.

Table 3 Estimated Krogh cylinder parameters at different anesthesia depths.

\begin{tabular}{lcccc} 
Anesthesia & $\mathrm{CMRO}_{2}\left(\mu \mathrm{mol} \mathrm{cm}^{-3} \mathrm{~min}^{-1}\right)$ fitted & $R_{\mathrm{t}}(\mu \mathrm{m})$ & $\mathrm{PO}_{2, \text { art }}(\mathrm{mmHg})$ fitted & $R_{\text {art }}(\mu \mathrm{m})$ fixed \\
\hline Alpha-chloralose & $1.56 \pm 0.36^{\mathrm{a}}$ & $106.5 \pm 8.9$ Fitted & $63.1 \pm 2.8$ & 9.1 \\
Alpha-chloralose + isoflurane & $1.38 \pm 0.35$ & $106.6 \pm 9.1$ Fitted & $66.0 \pm 2.9$ & 10.0 \\
Alpha-chloralose & $1.56 \pm 0.07$ & 106.5 Fixed & $63.0 \pm 2.0$ & 9.1 \\
Alpha-chloralose + isoflurane & $1.35 \pm 0.07$ & 106.5 Fixed & $64.9 \pm 2.0$ & 10.0 \\
\hline
\end{tabular}

astandard error.

at the same imaging location (160 $\mu \mathrm{m}$ deep) between the two anesthesia regimes. As expected, deeper anesthesia caused an increase in the baseline tissue $\mathrm{PO}_{2}$ [Fig. 4(c)]. The $\mathrm{PO}_{2}$ dependence on the radial distance from the arteriole was markedly less steep with the addition of isoflurane [Fig. 4(c)]. The measured $\mathrm{CMRO}_{2}$ decreased from $1.56 \pm 0.36 \mu \mathrm{mol} \mathrm{cm}{ }^{-3} \mathrm{~min}^{-1}$ (alphachloralose only) to $1.38 \pm 0.35 \mu \mathrm{mol} \mathrm{cm}{ }^{-3} \mathrm{~min}^{-1}$ (combined alpha-chloralose and isoflurane) (Table 3 ). The difference was not significant mostly due to the uncertainty in fitting the tissue radius $R_{\mathrm{t}}$. However, when $R_{\mathrm{t}}$ was treated as a fixed parameter in the $\mathrm{CMRO}_{2}$ estimation, which is likely to be appropriate here, considering that we are comparing $\mathrm{CMRO}_{2}$ measurements at the same location, the $\mathrm{CMRO}_{2}$ error decreased to $0.07 \mu \mathrm{mol} \mathrm{cm} \mathrm{cm}^{-3} \mathrm{~min}^{-1}$ (Table 3).

\section{Discussion}

Our study demonstrates that we can estimate $\mathrm{CMRO}_{2}$ using the Krogh cylinder model based on a single measurement modality-periarteriolar tissue $\mathrm{PO}_{2}$ measurement by two-photon microscopy in a single plane perpendicular to the vessel axis. With this method, no measurements of blood flow are required for the $\mathrm{CMRO}_{2}$ estimation. Using this method, we obtained a mean baseline $\mathrm{CMRO}_{2}$ of $1.71 \pm 0.16 \mu \mathrm{mol} \mathrm{cm}^{-3} \mathrm{~min}^{-1}$, within the error bounds of previously reported $\mathrm{CMRO}_{2}$ under similar anesthesia in rats measured by MRI $\left(2.5 \pm 1.0 \mu \mathrm{molcm}^{-3} \mathrm{~min}^{-1}\right){ }^{47}$

Several properties of the cortical vascular and cellular architecture make this simple model based on the Krogh cylinder applicable. First, the large tissue $\mathrm{PO}_{2}$ gradients around cortical penetrating arterioles (Figs. 1 and 2) are readily detectable with two-photon microscopy of $\mathrm{PO}_{2}$. Second, all oxygen consumed by the tissue adjacent to the penetrating arteriole is supplied by the arteriole. This is evident from (1) the large tissue $\mathrm{PO}_{2}$ gradients surrounding penetrating arterioles ${ }^{34,36,41,49-51}$ (Fig. 2) and (2) the absence of other oxygen sources (i.e., capillaries) in a 50 to $100-\mu \mathrm{m}$ radius around penetrating arterioles in rats [Fig. 1(b)] and also in mice. ${ }^{52}$ The oxygen delivery from penetrating arterioles is also consistent with observations of intravascular radial $\mathrm{PO}_{2}$ profiles in penetrating arterioles demonstrating a 
$\mathrm{PO}_{2}$ decrease from the vessel center to the arteriolar wall. ${ }^{41}$ Third, while at the cellular scale $\mathrm{CMRO}_{2}$ is likely heterogeneous, $\mathrm{CMRO}_{2}$ based on an approximate $\sim 200 \times 200 \mu \mathrm{m}^{2}$ area around a penetrating arteriole likely represents an accurate average $\mathrm{CMRO}_{2}$ estimate for a particular cortical depth. This is implied from the uniform distribution of neural and glial cells within a cortical column $(\sim 300 \mu \mathrm{m}$ in diameter in the rat primary somatosensory cortex) ${ }^{46}$ although this result does not necessarily guarantee that the density of synapses, which are the major contributors to the cortical oxygen consumption, ${ }^{53}$ is uniform as well. This also suggests that the spatial localization of our $\mathrm{CMRO}_{2}$ measurement is $\sim 200 \mu \mathrm{m}$. We also note that the depth resolution of $\mathrm{PO}_{2}$ imaging in our experiments is $\sim 5 \mu \mathrm{m} .{ }^{34}$ Fourth, the diameters of penetrating arterioles (15 to $20 \mu \mathrm{m}$ ) and their blood flow are sufficiently large so that the axial change in intravascular $\mathrm{PO}_{2}$ is small and thus, the influence of axial $\mathrm{PO}_{2}$ gradients is negligible. ${ }^{45,54}$ This is a requirement for the Krogh cylinder model, where a single cylindrical oxygen source supplies a homogeneous tissue cylinder. ${ }^{43}$

Here, we address several important aspects of our measurements, including differences from other reported measurements and potential sources of errors: (1) most of our measurements were obtained in the upper cortical layer (layer I, i.e., the top $150 \mu \mathrm{m}$ in the rat), which has a lower density of both neuronal cell bodies and synapses than most deeper cortical layers. ${ }^{55,56}$ Deeper cortical layers may exhibit higher $\mathrm{CMRO}_{2}{ }^{47}$ (2) The fit of Eq. (2) estimates $\mathrm{CMRO}_{2} /(D \alpha)$. Therefore, the accuracy of our $\mathrm{CMRO}_{2}$ estimate depends on the accuracy of the assumed values for the tissue permeability to oxygen (e.g., $D \alpha$ ). Unfortunately, the literature does not provide clear guidance on the accurate values to use for $D$ and $\alpha$ in the rat brain cortex. Measurements of $D$ have been typically preformed in muscle. In the hamster retractor muscle, Bentley et al. ${ }^{57}$ measured $D=2.41 \times 10^{5} \mathrm{~cm}^{2} \mathrm{~s}^{-1}$. The oxygen diffusion constant in water $\left(D=4 \times 10^{5} \mathrm{~cm}^{2} \mathrm{~s}^{-1}\right)$ that we applied in our calculations likely leads to an overestimation of $\mathrm{CMRO}_{2}$, although oxygen diffusion in the brain cortex is probably faster than in the muscle due to a higher water content in the brain cortex. ${ }^{58,59}$ On the other hand, the oxygen solubility in water $(\alpha=$ $1.3 \mu \mathrm{M} \mathrm{mmHg}^{-1}$ ) that we applied in our calculations is lower than the reported solubility in the frog sartorius muscle $\left(\alpha=1.6 \mu \mathrm{MmmHg}^{-1}\right),{ }^{60}$ and the solubility of oxygen in brain cortex may be even higher due to a higher lipid content. (3) The mean relative error of the $\mathrm{CMRO}_{2}$ estimates was $18 \%$, with the relative error of individual measurements varying between $12 \%$ and $23 \%$ (Table 1). The largest contributor to the error of the $\mathrm{CMRO}_{2}$ fit is the uncertainty in the $R_{\mathrm{t}}$ estimation. If we assume that oxygen flux through the arteriolar wall is $J_{\mathrm{O} 2}, \mathrm{CMRO}_{2}$ can be expressed by considering a simple mass balance of oxygen delivered to and consumed in the tissue cylinder as $\mathrm{CMRO}_{2}=2 R_{\mathrm{art}} J_{\mathrm{O} 2} /\left(R_{\mathrm{t}}^{2}-R_{\mathrm{art}}^{2}\right)$. If $J_{\mathrm{O} 2}$ is constant, the relative error of $\mathrm{CMRO}_{2}$ is related to the relative error of $R_{\mathrm{t}}$ as $\Delta \mathrm{CMRO}_{2} / \mathrm{CMRO}_{2} \approx-2 \Delta R_{\mathrm{t}} / R_{\mathrm{t}}$. Increasing the number of collected tissue $\mathrm{PO}_{2}$ points may be one possible approach to reduce this uncertainty. When performing the $\mathrm{CMRO}_{2}$ measurement on rat II (Fig. 2), we increased the number of acquired $\mathrm{PO}_{2}$ points by $2 \times$, which resulted in a significantly reduced relative error of both $R_{\mathrm{t}}(4.5 \%)$ and $\mathrm{CMRO}_{2}(11.6 \%)$. This approach, however, leads to doubling the data acquisition time. Our current acquisition of $\mathrm{PO}_{2}$ points on a grid pattern leads to a nonuniform distribution of $\mathrm{PO}_{2}$ points as a function of the radial distance from the arteriole. It is possible that other $\mathrm{PO}_{2}$ acquisition patterns, such as a radial grid, may improve the $\mathrm{CMRO}_{2}$ fitting error without the need to increase the number of $\mathrm{PO}_{2}$ measurement points. Future work will also investigate the effect of periarteriolar microvascular configurations in realistic vascular anatomical networks (VANs) on the periarteriolar tissue $\mathrm{PO}_{2}$ distribution and $\mathrm{CMRO}_{2}$ estimation. The combined experimental and numerical modeling approach ${ }^{61}$ may be utilized to further investigate the effect of nearby capillary and arteriolar vascular segments on the axial symmetry and radial extent of the periarteriolar tissue $\mathrm{PO}_{2}$ profiles, including potential changes of the tissue territories supplied by these vessels at different $\mathrm{CMRO}_{2}$ and $\mathrm{CBF}$ levels. ${ }^{41}$ The influence of the deviations of the $\mathrm{PO}_{2}$ measurement plane from a plane perpendicular to the arteriole on estimating $\mathrm{CMRO}_{2}$ may also require further attention. Finally, the $\mathrm{CMRO}_{2}$ fitting error is significantly lower if we fix $R_{\mathrm{t}}$ in the fitting procedure (Fig. 4). We believe that this approach may be appropriate when comparing $\mathrm{CMRO}_{2}$ changes at the same location.

Advances in the development of contrast agents for multiphoton imaging of $\mathrm{PO}_{2}$ and multiphoton imaging instrumentation may bring significant improvements and opportunities for $\mathrm{CMRO}_{2}$ measurements based on tissue $\mathrm{PO}_{2}$ maps. Significantly brighter and red-shifted $\mathrm{PO}_{2}$-sensitive dyes ${ }^{62}$ should allow faster, more accurate, and deeper tissue $\mathrm{PO}_{2}$ imaging in the brain cortex relative to our current $\sim 300-\mu$ m imaging depth limited primarily by the contamination of the deep signals with the out-of-focus phosphorescence. Faster and more accurate acquisition of tissue $\mathrm{PO}_{2}$ may significantly reduce both acquisition time and $\mathrm{CMRO}_{2}$ measurement error. It may potentially enable the possibility to estimate $\mathrm{CMRO}_{2}$ directly based on Eq. (1) both around penetrating arterioles and in a tissue away from them, providing smooth mapping of $\mathrm{PO}_{2}$ between capillaries. Currently, the complexity of the spatial distribution of the tissue $\mathrm{PO}_{2}$ around apparently randomly oriented cortical capillaries and the relatively small variation of the tissue $\mathrm{PO}_{2}$ around them are making it challenging to estimate $\mathrm{CMRO}_{2}$ based on Eq. (1) around capillaries. Alternatively, advanced modeling of oxygen advection and diffusion in realistic VANs may be utilized to estimate $\mathrm{CMRO}_{2}$ based on tissue $\mathrm{PO}_{2}$ imaging anywhere in the cortical tissue. ${ }^{32}$ In this approach, modeling may be further constrained by adding the blood flow and intravascular oxygenation measurements. ${ }^{63,64}$

\section{Conclusion}

The steady-state oxygen distribution in cortical tissue satisfies Poisson's equation, exhibiting a simple dependence on $\mathrm{CMRO}_{2}$ [Eq. (1)]. We present a method of estimating $\mathrm{CMRO}_{2}$ based on Eq. (1) that utilizes two-photon microscopy imaging of cortical tissue $\mathrm{PO}_{2}$ and exploits the specific morphology of cortical penetrating arterioles and neighboring microvessels that allows us to model the periarteriolar tissue $\mathrm{PO}_{2}$ distribution with the Krogh cylinder model. This method depends on a single imaging parameter (e.g., periarteriolar tissue $\mathrm{PO}_{2}$ ), making it significantly less complex and potentially more accurate than existing $\mathrm{CMRO}_{2}$ measuring methods that rely on multimodal imaging of blood oxygenation and blood flow. In addition, the measurement is spatially localized to a small tissue area $(\sim 200 \mu \mathrm{m})$, and it can be easily combined with other optical microscopy tools for preclinical studies in animals, providing approaches to address critical questions related to cortical pathological conditions and in understanding the fMRI signal. 


\section{Acknowledgments}

We gratefully acknowledge support from the National Institutes of Health (NIH) grants NS091230, NS55104, NS092986, NS057198, EB021018, EB00790, AG042026, and EB018464.

\section{References}

1. R. B. Buxton, "Interpreting oxygenation-based neuroimaging signals: the importance and the challenge of understanding brain oxygen metabolism," Front. Neuroenerg. 2, 8 (2010).

2. M. A. Mintun et al., "Brain oxygen utilization measured with O-15 radiotracers and positron emission tomography," J. Nucl. Med. 25(2), 177-187 (1984)

3. P. T. Fox et al., "Nonoxidative glucose consumption during focal physiologic neural activity," Science 241(4864), 462-464 (1988).

4. R. B. Buxton, Introduction to Functional Magnetic Resonance Imaging: Principles and Techniques, Cambridge University Press, Cambridge, United Kingdom (2002).

5. K. Uludağ et al., "Coupling of cerebral blood flow and oxygen consumption during physiological activation and deactivation measured with fMRI," Neuroimage 23(1), 148-155 (2004).

6. N. K. Logothetis, "What we can do and what we cannot do with fMRI," Nature 453(7197), 869-878 (2008).

7. D. A. Yablonskiy, A. L. Sukstanskii, and X. He, "Blood oxygenation level-dependent (BOLD)-based techniques for the quantification of brain hemodynamic and metabolic properties - theoretical models and experimental approaches," NMR Biomed. 26(8), 963-986 (2013).

8. T. Durduran et al., "Diffuse optical measurement of blood flow, blood oxygenation, and metabolism in a human brain during sensorimotor cortex activation," Opt. Lett. 29(15), 1766-1768 (2004).

9. N. Roche-Labarbe et al., "Noninvasive optical measures of CBV, StO (2), $\mathrm{CBF}$ index, and $\mathrm{rCMRO}(2)$ in human premature neonates' brains in the first six weeks of life," Hum. Brain Mapp. 31(3), 341-352 (2010).

10. X.-H. Zhu et al., "New opportunities for high-field in vivo MRS in studying brain bioenergetics and function," Brain Imaging Behav. 2(4), 232-241 (2008).

11. W. Chen et al., "Study of tricarboxylic acid cycle flux changes in human visual cortex during hemifield visual stimulation using $1 \mathrm{H}-\{13 \mathrm{C}\}$ MRS and fMRI," Magn. Reson. Med. 45(3), 349-355 (2001).

12. S.-H. Yee et al., "Quantitative measurement of oxygen metabolic rate in the rat brain using microPET imaging of briefly inhaled 15O-labelled oxygen gas," Nucl. Med. Commun. 27(7), 573-581 (2006).

13. D. S. F. Hyder et al., "Neurovascular and neurometabolic couplings in dynamic calibrated fMRI: transient oxidative neuroenergetics forblockdesign and event-related paradigms," Front. Neuroenerg. 2, 18 (2010).

14. Q. Shen, H. Ren, and T. Q. Duong, "CBF, BOLD, CBV, and $\mathrm{CMRO}_{2}$ fMRI signal temporal dynamics at 500-msec resolution," J. Magn. Reson. Imaging 27(3), 599-606 (2008).

15. W. Cui et al., "Non-invasive measurement of cerebral oxygen metabolism in the mouse brain by ultra-high field (17)O MR spectroscopy," J. Cereb. Blood Flow Metab. 33(12), 1846-1849 (2013).

16. X.-H. Zhu and W. Chen, "In vivo ${ }^{17} \mathrm{O}$ MRS imaging-Quantitative assessment of regional oxygen consumption and perfusion rates in living brain," Anal. Biochem. (2016).

17. A. Grinvald et al., "Functional architecture of cortex revealed by optical imaging of intrinsic signals," Nature 324(6095), 361-364 (1986).

18. J. P. Culver et al., "Diffuse optical tomography of cerebral blood flow, oxygenation, and metabolism in rat during focal ischemia," J. Cereb. Blood Flow Metab. 23(8), 911-924 (2003).

19. A. K. Dunn et al., "Spatial extent of oxygen metabolism and hemodynamic changes during functional activation of the rat somatosensory cortex," Neuroimage 27(2), 279-290 (2005).

20. G. Royl et al., "Hypothermia effects on neurovascular coupling and cerebral metabolic rate of oxygen," Neuroimage 40(4), 1523-1532 (2008).

21. J. Yi et al., "Visible-light optical coherence tomography for retinal oximetry," Opt. Lett. 38(11), 1796-1798 (2013).

22. S. P. Chong et al., "Cerebral metabolic rate of oxygen $\left(\mathrm{CMRO}_{2}\right)$ assessed by combined Doppler and spectroscopic OCT," Biomed. Opt. Express 6(10), 3941-3951 (2015).

23. B. Ning et al., "Ultrasound-aided multi-parametric photoacoustic microscopy of the mouse brain," Sci. Rep. 5, 18775 (2015).
24. J. Yao et al., "High-speed label-free functional photoacoustic microscopy of mouse brain in action," Nat. Methods 12(5), 407-410 (2015).

25. W. L. Rumsey, J. M. Vanderkooi, and D. F. Wilson, "Imaging of phosphorescence: a novel method for measuring oxygen distribution in perfused tissue," Science 241(4873), 1649-1651 (1988).

26. D. F. Wilson et al., "Effect of hyperventilation on oxygenation of the brain cortex of newborn piglets," J. Appl. Physiol. 70(6), 2691-2696 (1991).

27. M. Pawlowski and D. F. Wilson, "Monitoring of the oxygen pressure in the blood of live animals using the oxygen dependent quenching of phosphorescence," Adv. Exp. Med. Biol. 316, 179-185 (1992).

28. S. Sakadžić et al., "Simultaneous imaging of cerebral partial pressure of oxygen and blood flow during functional activation and cortical spreading depression," Appl. Opt. 48(10), D169 (2009).

29. A. K. Dunn et al., "Simultaneous imaging of total cerebral hemoglobin concentration, oxygenation, and blood flow during functional activation," Opt. Lett. 28(1), 28 (2003).

30. S. P. Chong et al., "Cerebral metabolic rate of oxygen $\left(\mathrm{CMRO}_{2}\right)$ assessed by combined Doppler and spectroscopic OCT," Biomed. Opt. Express 6(10), 3941-3951 (2015).

31. V. J. Srinivasan et al., "Depth-resolved microscopy of cortical hemodynamics with optical coherence tomography," Opt. Lett. 34(20), 3086-3088 (2009).

32. M. W. Dewhirst et al., "Determination of local oxygen consumption rates in tumors," Cancer Res. 54(13), 3333-3336 (1994).

33. O. S. Finikova et al., "Oxygen microscopy by two-photon-excited phosphorescence," ChemPhysChem 9(12), 1673-1679 (2008).

34. S. Sakadžić et al., "Two-photon high-resolution measurement of partial pressure of oxygen in cerebral vasculature and tissue," Nat. Methods 7(9), 755-759 (2010).

35. J. Lecoq et al., "Simultaneous two-photon imaging of oxygen and blood flow in deep cerebral vessels," Nat. Med. 17(7), 893-898 (2011).

36. A. Devor et al., "'Overshoot' of O2 Is required to maintain baseline tissue oxygenation at locations distal to blood vessels," J. Neurosci. 31(38), 13676-13681 (2011).

37. A. Devor et al., "Frontiers in optical imaging of cerebral blood flow and metabolism," J. Cereb. Blood Flow Metab. 32(7), 1259-1276 (2012).

38. A. Parpaleix, Y. G. Houssen, and S. Charpak, "Imaging local neuronal activity by monitoring $\mathrm{PO}_{2}$ transients in capillaries," Nat. Med. 19(2), 241-246 (2013).

39. S. M. S. Kazmi et al., "Three-dimensional mapping of oxygen tension in cortical arterioles before and after occlusion," Biomed. Opt. Express 4(7), 1061 (2013).

40. A. Devor et al., "Functional imaging of cerebral oxygenation with intrinsic optical contrast and phosphorescent probes," in Optical Imaging of Neocortical Dynamics, Vol. 85, B. Weber and F. Helmchen, Eds., pp. 225-253, Humana Press, Totowa, New Jersey (2014).

41. S. Sakadžić et al., "Large arteriolar component of oxygen delivery implies a safe margin of oxygen supply to cerebral tissue," Nat. Commun. 5, 5734 (2014).

42. D. G. Lyons et al., "Mapping oxygen concentration in the awake mouse brain," eLife 5, e12024 (2016).

43. A. Krogh, "The number and distribution of capillaries in muscles with calculations of the oxygen pressure head necessary for supplying the tissue," J. Physiol. 52(6), 409-415 (1919).

44. M. A. Yaseen et al., "Multimodal optical imaging system for in vivo investigation of cerebral oxygen delivery and energy metabolism," Biomed. Opt. Express 6(12), 4994-5007 (2015).

45. D. Goldman, "Theoretical models of microvascular oxygen transport to tissue," Microcirculation 15(8), 795-811 (2008).

46. P. S. Tsai et al., "Correlations of neuronal and microvascular densities in murine cortex revealed by direct counting and colocalization of nuclei and vessels," J. Neurosci. 29(46), 14553-14570 (2009).

47. P. Hermán, H. K. F. Trübel, and F. Hyder, "A multiparametric assessment of oxygen efflux from the brain," J. Cereb. Blood Flow Metab. 26(1), 79-91 (2005).

48. C. Lenz et al., "Local cerebral blood flow, local cerebral glucose utilization, and flow-metabolism coupling during sevoflurane versus isoflurane anesthesia in rats," Anesthesiology 89(6), 1480-1488 (1998).

49. B. R. Duling, W. Kuschinsky, and M. Wahl, "Measurements of the perivascular $\mathrm{PO}_{2}$ in the vicinity of the pial vessels of the cat," Pflügers Arch. 383(1), 29-34 (1979). 
50. E. Vovenko, "Distribution of oxygen tension on the surface of arterioles, capillaries and venules of brain cortex and in tissue in normoxia: an experimental study on rats," Pflügers Arch. 437(4), 617-623 (1999).

51. A. L. Vazquez et al., "Cerebral oxygen delivery and consumption during evoked neural activity," Front. Neuroenerg. 2, 11 (2010).

52. K. A. Kasischke et al., "Two-photon NADH imaging exposes boundaries of oxygen diffusion in cortical vascular supply regions," J. Cereb. Blood Flow Metab. 31(1), 68-81 (2011).

53. C. Howarth, P. Gleeson, and D. Attwell, "Updated energy budgets for neural computation in the neocortex and cerebellum," J. Cereb. Blood Flow Metab. 32(7), 1222-1232 (2012).

54. F. Kreuzer, "Oxygen supply to tissues: the Krogh model and its assumptions," Experientia 38(12), 1415-1426 (1982).

55. H. S. Meyer et al., "Number and laminar distribution of neurons in a thalamocortical projection column of rat vibrissal cortex," Cereb. Cortex 20(10), 2277-2286 (2010).

56. L. Anton-Sanchez et al., "Three-dimensional distribution of cortical synapses: a replicated point pattern-based analysis," Front. Neuroanat. 8, 85 (2014).

57. T. B. Bentley, H. Meng, and R. N. Pittman, "Temperature dependence of oxygen diffusion and consumption in mammalian striated muscle," Am. J. Physiol. 264(6 Pt 2), H1825-H1830 (1993).
58. A. M. Stewart-Wallace, "A biochemical study of cerebral tissue, and of the changes in cerebral Ædema," Brain 62(4), 426-438 (1939).

59. V. Rajanayagam, M. E. Fabry, and J. C. Gore, "In vivo quantitation of water content in muscle tissues by NMR imaging," Magn. Reson. Imaging 9(4), 621-625 (1991).

60. M. Mahler et al., "Reappraisal of diffusion, solubility, and consumption of oxygen in frog skeletal muscle, with applications to muscle energy balance," J. Gen. Physiol. 86(1), 105-134 (1985).

61. L. Gagnon et al., "Modeling of cerebral oxygen transport based on in vivo microscopic imaging of microvascular network structure, blood flow, and oxygenation," Front. Comput. Neurosci. 10, 82 (2016).

62. T. V. Esipova and S. A. Vinogradov, "Synthesis of phosphorescent asymmetrically $\pi$-extended porphyrins for two-photon applications," J. Org. Chem. 79(18), 8812-8825 (2014).

63. L. Gagnon et al., "Quantifying the microvascular origin of BOLD-fMRI from first principles with two-photon microscopy and an oxygen-sensitive nanoprobe," J. Neurosci. 35(8), 3663-3675 (2015).

64. L. Gagnon et al., "Multimodal reconstruction of microvascular-flow distributions using combined two-photon microscopy and Doppler optical coherence tomography," Neurophotonics 2(1), 015008 (2015).

Biographies for the authors are not available. 